\title{
Anaesthesiology directors: more support on the way as Association of Anesthesia Clinical Directors goes international
}

\begin{abstract}
Management and leadership issues are becoming ever more important to anaesthesiologists, in particular those with special responsibility for anaesthesia, pain, intensive care and operating theatre services. Tomorrow's anaesthesiologists will need to be increasingly competent in management and leadership. What lies behind this trend?

One driving force is the quest for medical quality. In the UK, government and professional regulatory bodies have had to restore shattered public confidence in the medical profession, following high-profile catastrophic failures of medical quality, such as the Bristol inquiries into paediatric heart surgery mortality $[1,2]$ and the recent case of a murderous single-handed general practitioner [3]. Their responses have pushed clinical performance right into the spotlight, across the whole spectrum of medical care. 'Clinical governance', an initiative introduced in a 1998 government white paper [4], makes clinical performance a corporate responsibility for the first time for hospital boards of directors. No longer is it a matter solely for the profession. The clinical governance initiative also paves the way towards increasingly demanding responsibilities for doctors, relating for example to risk management, poorly performing doctors and tools for quality improvement such as the effective use of clinical audit.
\end{abstract}

A second major force behind the increasing profile of management in anaesthesia is the quest for efficiency [5]. Perennial problems, for example of productivity on a tight budget, or of matching suitable cases with suitable human resources, are now getting far more help from the management sciences. Economic analyses about the costs of anaesthesia services $[6,7]$ are available to help clinical directors with their decision making on operating theatre profitability and expenditure; whilst techniques drawn from industrial operations management are being applied to problems of optimization and forecasting in operating theatres $[8,9]$.
At the sharp end of clinical governance, with the responsibility for implementing all the consequent changes, will be the anaesthesia clinical director. Yet a recent survey of this group in the UK suggested that not all were well prepared or adequately supported to enable them to deal with the increasingly challenging and exposed role involved. Less than half of those surveyed had clear instructions from those they reported to about the tasks they were to carry out; most were under-funded; and only a minority had access to the information support they needed to underpin decision making. As a group, they were overloaded with work, under-prepared for the role, and under-networked in terms of external support or training opportunities[10]. Previous writings about the work of anaesthesia clinical directors also painted a picture of unmet training needs and a perceived gap in terms of networks and support structures [11,12].

A small but vital group of anaesthesiologists, who originally got together to remedy things in the USA, formed the Association of Anesthesia Clinical Directors. The Association has been in existence since 1988, aiming to create a forum for clinical directors and those interested in management in anaesthesia, to hold educational events, to take forward the interests of this important group of anaesthesiologists and to encourage development of this new subspecialty. AACD has two conference events each year, and also a discussion group accessed via the Association's web page, which encourages discourse on management issues. The main conference is an annual workshop on Operating Room management, held each Spring in the Western USA.

Until recently the focus for AACD was its wholly US-based membership. However, over the last few years, more and more international delegates have been finding their way independently to the OR Management Workshop. These anaesthesiologists clearly all share the belief that networking, and encouraging 
discussion from an international perspective is a useful way of exploring key issues in management, and can be a pathway towards finding some of the solutions needed to manage complex anaesthesia services.

On the evidence so far, it seems that the problems that clinical directors face are not unique to individual countries. Examples of lessons that have been rated as highly transferable from one country to another include: scheduling in operating rooms; benchmarking in anaesthesia; microcostings, cost minimization and the true costs of new programmes; motivation; management of change; and looking ahead in information and IT. Other key management-of-anaesthesia issues, which at face value are of international relevance, might include: clinical leadership; professional selfregulation; effectiveness of medical care; ensuring efficiency and profitability in anaesthesia services; learning from critical incidents, and obtaining sound information to support operational and strategic decision making.

Anaesthesiologists are increasingly aware of the value of sharing lessons and solutions, but networking between hospitals can be difficult and costly, and between countries even more so. The growth in international interest prompted AACD to extend beyond its home base and set up an international initiative building on its 11-year experience of serving the needs of managerially oriented anaesthesiologists.

The first step has been to design the OR Management Workshop, held in February 2000 in San Francisco, so as to be relevant to a wide range of international delegates. Shortly afterwards, in June 2000, the first International Network meeting of AACD will be launched at the 12th World Congress of Anaesthesiologists (WCA) in Montreal, Canada, with the kind support of the World Federation of Societies of Anaesthesiologists. Lectures on OR management, change and the culture of hospitals, will be followed by a discussion session taking on board the views of delegates on the best way forward in terms of collaboration between clinical directors internationally.

Anaesthesiologists who are interested in management, or for whom management is an important part of the job, now have an opportunity to shape the future and develop their own network. Not all those potentially interested will be attending the WCA, but the website and discussion page (http:// www.aacdhq.org) is open to all.

\section{Dr Gavin Thoms}

Manchester Royal Infirmary, Manchester M13 9WL, UK

Note: Anaesthesiologists wishing to attend the first free-access AACD International Network meeting to be held at $2 \mathrm{pm}$ on 4 th June 2000, at the Montreal Convention Center, should register interest by e-mail (gavin.thoms@man.ac.uk).

\section{References}

1 Smith R. All changed, changed utterly (editorial). $\mathrm{Br} \mathrm{Med}$ J 1998; 316: 1917-1918.

2 Dobson R. Bristol Inquiry opens (News). Br Med J 1999; 318: 754.

3 Jenkins R, Duce R. Britain's worst serial killer. The Times 2000; 1st February: 1-2.

4 Department of Health. A First Class Service: Quality in the New NHS. London: Department of Health, 1998.

5 Audit Commission. Anaesthesia Under Examination. London: Audit Commission 1997.

6 Macario A, Vitez TS, Dunn B, McDonald T. Where are the costs in Perioperative Care? Anesthesiology 1995; 83: 1138-1144.

7 Dexter F, Macario A. Does early extubation ('fast-tracking') of coronary artery bypass graft surgery decrease perioperative costs? Appropriate analysis of direct variable costs. Anesthesiology 1997; 87: 181-182.

8 Dexter F, Macario A. Applications of Information Systems to Operating Room Scheduling. Anesthesiology 1996; 85: 1232-1234.

9 Dexter F, Macario A, Qian F, Traub RD. Forecasting Surgical Groups' Total Hours of Elective Cases for Allocation of Block Time. Anesthesiology 1999; 91: 1501-1508.

10 Thoms GMM, McHugh GA, Pollard BJ, Moore J. Anaesthesia Clinical Directors in the United Kingdom: organisation, objectives and support needs. Anaesthesia 1999; 54: 753-760.

11 BAMM. Leading Clinical Services: the Evolving role of the Clinical Director. Cheadle, UK: British Association of Medical Managers, 1997.

12 Simpson J. BAMM Clinical Director's survey. Clinician Management 1993; 2: 13-15. 